\title{
The physics and astrophysics of Type Ia supernova explosions
}

\author{
Mike Guidry $^{1,2,3, *}$, Bronson Messer ${ }^{4,1, \dagger}$ \\ ${ }^{1}$ Department of Physics and Astronomy, University of Tennessee, Knoxville, TN 37996, USA \\ ${ }^{2}$ Physics Division, Oak Ridge National Laboratory, Oak Ridge, TN 37830, USA \\ ${ }^{3}$ Computer Science and Mathematics Division, Oak Ridge National Laboratory, Oak Ridge, TN 37830 , USA \\ ${ }^{4}$ National Center for Computational Sciences, Oak Ridge National Laboratory, Oak Ridge, TN 37831, USA \\ E-mail:*guidry@utk.edu, †bmesser@ornl.gov \\ Received March 8, 2013; accepted March 16, 2013
}

A supernova is a transient astronomical event of spectacular peak brightness that is associated with an exploding star. Supernovae exhibit a range of observational characteristics that historically has led to a rich set of classifications and sub-classifications. Despite the complexity of the observationally-based supernova taxonomy, we now believe that all supernovae are caused by just one of two basic mechanisms: (i) the collapse of the core of a massive star late in its life, or (ii) a runaway thermonuclear explosion in a white dwarf. The former is termed the core-collapse mechanism, and is powered by gravitational energy; the latter is termed the thermonuclear mechanism, and is powered by the release of nuclear binding energy under explosive thermonuclear burning conditions. The papers in this issue of Frontiers of Physics are concerned with a particular type of supernova that is classified observationally as a Type Ia supernova (which we shall often abbreviate by SNe Ia). A Type Ia supernovae is identified by the shape of its lightcurve (the observed brightness as a function of time), the presence of spectral lines associated with heavier elements like silicon or calcium, but the absence of spectral lines associated with hydrogen and helium (which is quite surprising, since $\mathrm{H}$ and He are the most abundant elements in the Universe).

The mechanism of Type Ia supernova explosions is of both practical and fundamental interest for the disciplines of physics, astrophysics, and cosmology. The greatest practical importance has been for cosmology, because SNe Ia events provide a unique means to measure very large distances in the Universe. A standard candle is a light source that always has the same intrinsic brightness; thus standard candles permit distance measurement by comparing the observed brightness of an event with the standard brightness. Different SNe Ia have similar but not identical lightcurves and are not standard candles. However, because the lightcurves of different Type Ia events are found to be closely related, empirical methods that depend only on observations allow their lightcurves to be collapsed approximately to a single curve $[1,2]$. Thus, Type Ia supernovae are termed standardizable candles, and once standardized they become powerful tools to measure distances. (Note, however, that recent

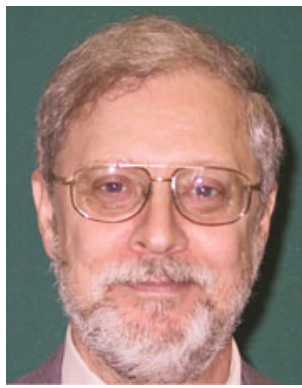

mW Amidn

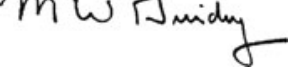

Prof. Mike Guidry

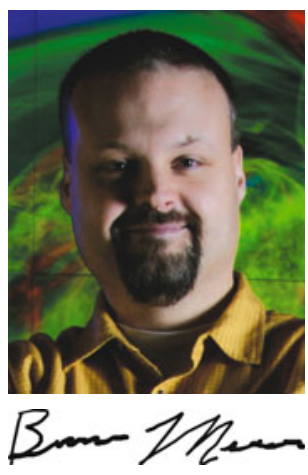

Prof. Bronson Messer results have suggested that substantial subclasses of SNe Ia may not be as homogeneous as originally thought; the paper by Hillebrandt et al. [3] in the current issue discusses this issue.)

Type Ia standardizable candles are particularly valuable because their extreme brightness makes them visible at very large distances compared with most standard candles of use in astronomy. The most spectacular outcome of studying SNe Ia at large distances (more precisely, at large redshift) has been the realization that the expansion of the Universe appears to be accelerating. Within the standard interpretation of modern cosmology, that acceleration is ascribed to a mysterious dark energy that pervades the Universe at very low density but accounts for some $70 \%$ of the mass-energy of the Universe because it is ubiquitous on cosmological scales. It effectively causes gravity to become repulsive when inserted in the field equations of general relativity (see Refs. $[4,5]$ for reviews). The awarding 
of the 2011 Nobel Prize in Physics to Saul Perlmutter, Brian Schmidt, and Adam Riess as the leaders of the teams responsible for using Type Ia supernovae as tools to establish the acceleration of the cosmic expansion is testament to the significance of this surprising result. More generally, as a consequence largely of the calibrated distance scales deriving from Type Ia supernova observations, and the determination of the dark energy density and a number of other cosmological parameters from precise and systematic study of fluctuations in the cosmic microwave background (which was acknowledged by another Nobel Prize awarded to John Mather and George Smoot in 2006), cosmology was transformed in hardly more than a decade from a notoriously qualitative and poorly-constrained discipline to one based increasingly on precise measurements yielding stringent tests of its predictions.

These developments have motivated implementation of increased capability to observe Type Ia supernovae. It may be estimated that in a large galaxy such as our own there is on average a SNe Ia event about once every 50-100 years (the supernovae of Tycho in 1572 and Kepler in 1604 were the most recently observed confirmed SNe Ia events in the Milky Way). However, the observation rate is much higher if we can monitor many galaxies at once. Modern supernova searches repeatedly image patches of sky containing thousands of galaxies. Pixel by pixel subtraction of images obtained for the same patch of sky but for times separated by days or weeks then reveals the sudden changes in brightness characteristic of a supernova explosion. Telescopic studies of these candidate regions then can confirm whether a supernova has been observed and its type. Implementation of such technology currently permits the detection of hundreds of SNe Ia events per year in distant galaxies.

Even leaving aside the practical role of Type Ia supernovae in the revolution that cosmology has undergone, SNe Ia events are remarkable from a purely physics and astrophysics perspective. They are among the most violent explosions in the Universe, releasing of order $10^{51} \mathrm{ergs}\left(10^{44} \mathrm{~J}\right)$ of energy on a timescale of a second or so from a region of planetary size (this is about 18 orders of magnitude more energy than the Sun emits in one second), and can outshine an entire galaxy of normal stars when at their peak light output. This begs for an explanation in terms of fundamental physics and astrophysics. Because of the gigantic energy release in a small region over a very short period of time, the conditions in a Type Ia explosion are extreme. Simulations indicate that temperatures in the hottest zones can approach $\sim 10^{10} \mathrm{~K}$, with densities as large as $\sim 10^{9} \mathrm{~g} \cdot \mathrm{cm}^{-3}$, and the temperature may change at rates as high as $\sim 10^{17} \mathrm{~K} \cdot \mathrm{s}^{-1}$. A quantitative understanding of the Type Ia explosion presents formidable physics and computational issues that are difficult to deal with in the large numerical simulations that are required to model such events. The aforementioned extremes in temperature and density, along with the prodigious gravitational fields in which the thermonuclear explosion progresses produce a problem in turbulent reactive flow that is distinctly different from those encountered in terrestrial laboratories. Although in broad outline we believe that we know many of the basics underlying this mechanism, our understanding lags for many important details.

The data constraining our physical understanding of the SNe Ia mechanism come primarily from lightcurves, spectra, composition and velocity of the expanding debris inferred from spectroscopy (e.g., see the papers in the present volume by Hillebrandt et al. [3] and Höflich et al. [6]), and more general astronomical observations that elucidate the environments in which explosions are observed (see the paper by Calder et al. [7] in the present issue that examines how explosions are influenced by properties of progenitor systems in their galactic environments). These data are reasonably well explained by the assumption that Type Ia explosions correspond to the complete incineration of a carbon-oxygen white dwarf component of a binary star system because of a thermonuclear runaway that burns the carbon and oxygen to isotopes of maximal thermodynamic stability in the vicinity of iron and nickel (commonly termed the iron group of nuclei) and other intermediate-mass elements, under conditions that are initially highly electron-degenerate. (The pressure in the white dwarf equation of state is dominated by electrons that occupy the lowest available quantum states; such electron configurations are termed degenerate.) Thus, these are thermonuclear supernovae, unlike all other known classes of supernovae, which are thought to be powered gravitationally by core collapse of a massive star.

Despite rather general agreement on the mechanism outlined in the preceding paragraph, there remain many unanswered questions associated with both the astrophysics and the physics of this process. Chief among them is that observational astronomy has so far not been able to give a definitive answer to the question of what initiates such gargantuan explosions (see Ref. [8] for a concise review). The two answers that have achieved the most traction thus far have been (i) the single-degenerate mechanism, where the explosion is thought to occur because of accretion from a more normal (typically main sequence or giant) star onto a white dwarf in a binary system, and (ii) the double-degenerate mechanism, where the explosion is thought to result from a merger of two white dwarfs in a binary system. Other possibilities, such as the sub-Chandrasekhar mass mechanism, in which a thermonuclear runaway is initiated in the outer accreted helium layers of the white dwarf that drives a detonation wave inward, also have 
been suggested as possibilities for at least some Type Ia explosions. Although the single-degenerate mechanism is probably favored by a majority of researchers, none of these scenarios appear completely consistent with observations at this point as the sole source of Type Ia explosions. This invites speculation that there may be more than one population of Type Ia supernovae, all corresponding to thermonuclear runaways in white dwarf material but with each population representing a different mechanism for initiation of the explosion. Despite this uncertainty, much of the physics addressed in this volume might be quite similar for either the single degenerate (accretion on a white dwarf) or double degenerate (merger of two white dwarfs) pictures, since the distinction between the two may be largely one of initial conditions rather than the dynamics of the explosion itself. In any of the plausible Type Ia mechanisms the explosion is driven by a thermonuclear runaway in white dwarf matter.

In the Type Ia explosion there is a thermonuclear flame front corresponding to conversion of carbon and oxygen fuel into heavier elements by nuclear reactions that releases large amounts of nuclear binding energy. This explosion is extremely violent since it is initiated under highly-degenerate conditions (which leads to a positive-feedback thermonuclear runaway), and involves energy and temperature scales far beyond our everyday experience. However, it shares many qualitative properties with ordinary chemical burning. There is a flame front that proceeds through the white dwarf material, with "cooler" (a highly relative term in this context!) unburned fuel in front and hot burned products (ash) behind. The thermonuclear flame associated with the burning front can be remarkably localized - as narrow as millimeters. (That it is so narrow follows largely from the extremely strong temperature dependence of the carbon burning that initiates the thermonuclear runaway.) Thus there are two very different distance scales characterizing the explosion: the size of the white dwarf, which is of order $10^{4} \mathrm{~km}$, and the width of the flame that consumes it, which typically is many orders of magnitude smaller. There also are highly disparate time scales to contend with, since the nuclear burning times can vary over many orders of magnitude and these can in turn be orders of magnitude faster than the characteristic timescale for response of the fluid. This presents severe difficulties in accurately modeling Type Ia explosions, since standard numerical techniques have difficulty addressing such disparate scales in space and time. A common approach (borrowed from the engineering disciplines concerned with the same kinds of turbulent chemical reactive flow) in dealing with the spatialscale disparity utilizes a subgrid model to describe the flame front, while treating the larger-scale fluid motion by a hydrodynamics approximation, producing a so-called large-eddy simulation (LES). A crucial issue is then the physics of the thermonuclear burning entering into such a subgrid model. The nature of this subgrid is determined by the mode of thermonuclear burning.

In both thermonuclear and ordinary chemical burning there is an important distinction associated with the speed of the flame front. If the flame front advances through the fuel at a speed less than the local speed of sound in the medium (subsonic), it is termed a deflagration wave. In a deflagration, fuel in front of the advancing flame is heated to ignition temperature by conduction of heat across the flame front. (Matter described by a degenerate equation of state is a very good thermal conductor, much like a metal.) If the flame front is thin enough so that the turbulent eddies (both those produced by the propagation of the flame front itself or those present in the star from other sources) serve only to "wrinkle" the front, the front is said to be a flamelet. Considerable effort has been expended in the past two decades in formulating effective subgrid models for flamelet propagation in SNe Ia [9, 10]. On the other hand, if the flame front advances at a speed greater than that of sound in the medium (supersonic) it is called a detonation wave. In a detonation a shock wave forms and the fuel in advance of the flame is heated to ignition temperature by shock heating. The paper by Parete-Koon et al. [11] in this issue surveys some important aspects of our present understanding of the detonation flame and how it propagates, and thus of microscopic physics input to possible subgrid models for this mode of burning.

Deflagrations and detonations produce different isotopic abundance signatures in the ash that is left behind. The detailed observational characteristics of Type Ia supernovae (in particular, the elemental abundances and velocities detected spectroscopically in the expanding debris) could be accounted for most naturally if we assume that part of the burning proceeds as a deflagration and part of it as a detonation. This is a difficulty for the theory because general considerations suggest that the explosion starts off as a deflagration and it is not easy for computer simulations in astrophysical environments to get the burning to transition to a detonation without invoking assumptions having only loose justification at our present level of understanding. Thus, we believe that the proposed SNe Ia mechanism is plausible in outline, but there are bothersome details that leave some doubt about whether we understand fully the burning that powers these spectacular explosions.

The SNe Ia explosion is initiated by carbon burning, which quickly raises the temperature and initiates burning of oxygen and the varied reaction products that result from carbon and oxygen burning. The rapid temperature rise is associated with coupling of the large energy release from the thermonuclear burning described by the reaction 
network to the fluid of the white dwarf, which is described by hydrodynamics. As alluded to above, this coupling introduces substantial computational challenges because of the disparity of the various physical timescales that characterize the thermonuclear network and fluid motion, which can easily range over 10-20 orders of magnitude. Under these conditions, as the thermonuclear flame burns through the white dwarf the carbon and oxygen fuel in each region is converted quickly into hundreds of new isotopes. The entire white dwarf is consumed on a timescale of order seconds, with the burned and unburned material violently ejected by the explosion, leaving no detectable remnant.

Thus a crucial aspect of the physics concerns the energy released and the new isotopes synthesized in a Type Ia supernova explosion. If the explosion achieves nuclear statistical equilibrium (NSE), basic thermodynamic considerations and the known binding energies dictate that the primary final products of this burning will be isotopes in the vicinity of iron and nickel (the iron group). However, observations find substantial populations of elements intermediate in mass between carbon-oxygen and the iron group with high velocities in the outer layers of the expanding debris, indicating that not all zones burn to NSE. This suggests that the burning is partially by deflagration and partially by detonation, as proposed above. Loosely the argument is that if the white dwarf is consumed by a detonation wave the fuel does not have time to expand before being burned, and high-temperature burning at white dwarf densities tends to produce NSE because the nuclear reaction rates are very large under those conditions. However, if a slower deflagration wave can partially expand material before a detonation wave is produced, the burning in the expanded material will involve lower average reaction rates and need not go completely to NSE, thus producing irongroup nuclei but also substantial amounts of intermediate-mass isotopes.

The most realistic simulations of Type Ia supernovae done to this point have employed only very small kinetic networks to describe the thermonuclear burning that powers the event, because standard methods for solving the kinetic equations have not been fast enough to couple more realistic networks to the hydrodynamical simulation. Such networks are a caricature of the thermonuclear network that is operative in a realistic explosion, where hundreds of isotopes are produced in significant quantities. Recent advances in the solution of larger, more realistic networks coupled to multidimensional hydrodynamics provide some hope that a more physically-correct treatment of the network powering the explosion may now be possible.

Such more realistic networks are important not only for the energy release powering the event, but also for understanding the distribution of the large number of new isotopes produced in the expanding debris of the explosion. Some of these developments are reviewed in the paper by Travaglio and Hix [12] contained in this issue, and in Ref. [13].

Finally, let us circle back to the issue of cosmology. In addition to the intrinsic interest in understanding such spectacular events, a detailed and systematic understanding of the physics and astrophysics underlying the Type Ia mechanism could lead to more precise tests of cosmology than are currently possible. Because the cosmic fluid is of such low density (corresponding on average to just a few hydrogen atoms per cubic meter of space), the equation of state governing the Universe on cosmological scales is expected to take the simple linear form $P=\sum_{i} w_{i} \varepsilon_{i}$, where $P$ is the pressure, $i$ labels independent components of the cosmic fluid, $\varepsilon_{i}$ is the energy density, and the parameter $w_{i}$ determines the nature of the $i$ th component of the cosmic fluid. Although this is a very simple equation of state, the actual values of $w_{i}$ have profound implications for the history of the Universe. Causality limits $w_{i}$ to values less than one, massless particles (radiation) have $w_{i}=1 / 3$, and massive particles have $w_{i}=0$. Any component of the cosmic fluid having $w_{i}<-1 / 3$ is termed dark energy; the contribution of such a component causes the cosmic expansion to accelerate rather than decelerate.

The calibrated distance scales derived from empirically-corrected SNe Ia lightcurves indicate that the Universe is presently accelerating and dominated by a (dark-energy) component with $w_{i} \leqslant-1 / 3$. However, the scatter of observational data points around the standardized lightcurves does not permit $w_{i}$ for this component to be determined with sufficient precision to distinguish among values predicted by different theories of dark energy, or to infer if it changes with time. Improved observations and improved understanding of the lightcurve calibration would permit a more precise determination of $w_{i}$, and thus permit quantitative tests of various theories of dark energy. Hence, a better understanding of the physics and astrophysics underlying the empirical standardizable candle correction through a detailed understanding of the Type Ia explosion mechanism and its connection to the corresponding observational lightcurves and spectral information could have large implications for cosmology. The paper by Hillebrandt et al. [3] contained in this issue provides a measure of where we stand with respect to comprehensive, multiphysics simulations of Type Ia events in comparison with available observational data. We may hope that this and the other papers of this volume represent a step toward a more detailed and fundamental 
understanding of the SNe Ia mechanism, both for its intrinsic interest and for its potential applications in precision cosmology.

\section{References}

1. A. Kim, LBNL Rep. No. LBNL-5616, 2004

2. M. Hamuy, M. M. Phillips, N. B. Suntzeff, R. A. Schommer, et al., Astron. J., 1996, 112: 240

3. W. Hillebrandt, M. Kromer, F. K. Röpke, and A. J. Ruiter, Front. Phys., 2013, 8(2): 116

4. J. A. Frieman, M. S. Turner, and D. Huterer, Annu. Rev. Astro. Astrophys., 2008, B46(1): 385

5. A. Goobar1 and B. Leibundgut, Annu. Rev. Nucl. Part. Sci., 2011, 61: 251

6. P. Höflich, P. Dragulin, J. Mitchell, B. Penney, B. Sadler, T.
Diamond, and C. Gerardy, Front. Phys., 2013, 8(2): 144

7. A. C. Calder, B. K. Krueger, A. P. Jackson, and D. M. Townsley, Front. Phys., 2013, 8(2): 168

8. D. A. Howell, Nat. Commun., 2011, 2: 350

9. A. Khokhlov, Astrophys. J., 1995, 449: 695

10. W. Schmidt, J. C. Niemeyer, W. Hillebrandt, and F. K. Röpke, Astron. Astrophys., 2006, 450(1): 283

11. S. T. Parete-Koon, C. R. Smith, T. L. Papatheodore, and O. E. B. Messer, Front. Phys., 2013, 8(2): 189

12. C. Travaglio and W. R. Hix, Front. Phys., 2013, 8(2): 199

13. M. Guidry, J. Comp. Phys., 2012, 231: 5266, arXiv: 1112.4778 